Research Article

\title{
Efficacy of Piroxicam Mesotherapy in Treatment of Knee Osteoarthritis: A Randomized Clinical Trial
}

\author{
Hamid Reza Farpour (D), ${ }^{1,2}$ Fahime Estakhri $\mathbb{D}^{0},{ }^{3}$ Mohadese Zakeri, ${ }^{3}$ and Reyhaneh Parvin ${ }^{3}$ \\ ${ }^{1}$ Shiraz Geriatric Research Center, Department of Physical Medicine and Rehabilitation, Shiraz University of Medical Sciences, \\ Shiraz, Iran \\ ${ }^{2}$ Bone and Joint Diseases Research Center, Shiraz University of Medical Sciences, Shiraz, Iran \\ ${ }^{3}$ Department of Physical Medicine and Rehabilitation, Shiraz University of Medical Sciences, Shiraz, Iran
}

Correspondence should be addressed to Fahime Estakhri; fahime.estakhri@gmail.com

Received 25 April 2020; Revised 6 June 2020; Accepted 17 July 2020; Published 3 August 2020

Academic Editor: Mario Ledda

Copyright (C) 2020 Hamid Reza Farpour et al. This is an open access article distributed under the Creative Commons Attribution License, which permits unrestricted use, distribution, and reproduction in any medium, provided the original work is properly cited.

\begin{abstract}
Introduction. Knee osteoarthritis (KOA) is one of the most common degenerative diseases that lead to pain and disability. Oral NSAIDs are effective drugs used to alleviate symptoms in patients with KOA, but they have several important complications, especially in the elderly. In this study, we evaluated the effectiveness of mesotherapy on pain reduction and improvement of functioning in patients with KOA. Methods. Sixty-two patients with KOA, grade 2-3 of the Kellgren-Lawrence scale, were randomized into two groups: the mesotherapy group, in which two injections were applied with piroxicam at a 10-day interval, and the oral group, in which piroxicam was prescribed for 10 days. The patients were evaluated before the treatment and 2, 4, and 8 weeks after it using the Visual Analogue Scale (VAS), Oxford Knee Scare (OKS), and Western Ontario McMaster University Osteoarthritis Index (WOMAC, Persian version). Results. There was no significant difference in demographic characteristics and baseline pain and function scores between the two groups. After 2, 4, and 8 weeks of follow-up, VAS, WOMAC, and OKS scores significantly improved in both groups (in the mesotherapy group: $p$ value $<0.001$ in all three scores and in the oral group: $p$ value $<0.001$ in the VAS scale and $p$ value $<0.05$ in WOMAC and OKS scores). There was no significant difference between the two groups at any time in the VAS score, but improvement in WOMAC and OKS scales in the mesotherapy group was significantly better ( $p$ value $<0.05$ in both scales [ $p$ value $<0.03$ in OKS and $p$ value $<0.02$ in WOMAC scales]). Side effects in both groups were not serious: limited heart burn in $32.2 \%$ of the total subjects in the oral group and pain at the injection site in $3.2 \%$ and bruises in $38.7 \%$ of the total subjects in the mesotherapy group. Conclusion. Mesotherapy is an effective and safe treatment modality in patients with mild-to-moderate KOA in the short term. This trial is registered with IRCT2017052434113N1.
\end{abstract}

\section{Background}

Knee osteoarthritis (KOA) is one of the most common chronic joint diseases. Due to aging, increase in life expectancy, and epidemic of obesity, incidence and prevalence of $\mathrm{KOA}$ is rising [1]. According to the survey performed in the United States, the prevalence of radiologic KOA is about $20-28 \%$, and in symptomatic KOA, it is about $7-17 \%$ in people over 45 years [2]. Pain and loss of function are the main symptoms that lead to treatment [1]. KOA incurs significant costs economically and socially [3]. Arthritis, after headache and back lumbago, is the third common pain condition that causes loss of productive work time in the United States [4].

Pain and loss of function are the main symptoms that lead to treatment [1]. There are different nonsurgical and surgical methods for decreasing pain, improving function, and preventing or correcting deformities in KOA [5].

In conservative management, nonpharmacological options are education, exercise, weight loss, physical therapy, acupuncture, assistive devices, taping, etc. Pharmacological treatment options are topical agents (capsaicin and diclofenac sodium), systemic drugs (acetaminophen, nonsteroidal anti-inflammatory drugs [NSAIDs], opioids, and 
duloxetine), and localized injection therapy (peri- and intraarticular prolotherapy, PRP, hyaluronic acid, glucocorticoids, and ozone) [5-13].

One of the important challenges in the KOA management is the most effective and safe treatment for each patient [14]. Systemic NSAIDs are effective, low cost, and easy access drugs for pain relief and functional improvement in patients with KOA, but they have several side effects such as gastrointestinal and cardiovascular problems. The risk of complications on the presence of other risk factors such as aging is higher [15]. However, newer NSAIDs (for example: lornoxicam) have better effects on pain and function with lesser complications in patients with KOA [16].

Mesotherapy is a procedure used to inject pharmacological substances or plant extracts into the dermis or subcutaneous layer of the skin. This technique was invented by Pistor in 1958 for the treatment of musculoskeletal pain and vascular and infectious diseases [17-19]. "One of the main advantages of mesotherapy is that local pharmacological effects can be obtained without the need for high systemic concentration. Mesotherapy in combination with systemic therapy can produce synergistic effects" [19, 20]. Side effects relative to systemic therapy can be reduced due to local injection of drugs. Adverse effects of mesotherapy depend on the technique and active substance, such as allergic reactions, pain, ecchymosis, bleeding, and infection. These side effects are often transitory and reversible $[18,19]$.

Mesotherapy is a type of alternative therapy used to control the local pain [21]. In one study, this method reduced cervical and low back pain by at least $50 \%$ compared to the baseline [19]. In another study, mesotherapy with NSAIDs and corticosteroids in acute low back pain could provide the same therapeutic effects as that induced by systemic drug administration [22]. In one study, about pes anserine bursitis in symptomatic osteoarthritis patients, mesotherapy with diclofenac reduced pain and ultrasonographic findings significantly [20]. In a study on patients with KOA, mesotherapy with different protocols depending on the acute or chronic phase of OA in six special points around the knee was done and compared with oral administration of diclofenac for three months. Both treatments improved the patients' clinical conditions, but mesotherapy was more efficient in WOMAC scores with fewer side effects [17].

There are few surveys and specific protocols for treatment of KOA symptoms with mesotherapy. Thus, we aimed to compare the efficacy of mesotherapy with piroxicam and short-term prescription of this drug on decreasing pain and improving the functions of patients with KOA.

\section{Materials and Methods}

2.1. Trial Design. This study was a single-blinded, randomized clinical trial with a blocked randomization and parallel design. The consort flowchart is illustrated in Figure 1.
2.2. Participants. Participants in this study were selected from patients with knee pain that referred to physical medicine and rehabilitation clinics in Shahid Rajaee, Shahid Faghihi, Imam Reza, and Hafez hospitals (academic centers of the Shiraz University of Medical Sciences [SUMS]) between September 2017 and January 2018.

2.3. Selection Criteria. The inclusion criteria were clinical symptoms of KOA (based on the clinical criteria of the American College of Rheumatology [1]) with a symptom duration of more than 3 months, grade 2 or 3 (mild or moderate) of the Kellgren-Lawrence scale in X-ray [23] within the past 1 month, age of 40-70 years in both sexes, Visual Analogue Scale of more than three [24], absence of any pathologic conditions around the knee such as bursitis or cellulitis, and willingness to participate in the study. Exclusion criteria included any intra- or periarticular injection during the past three months; use of NSAIDs for pain relief during the past three days; a history of cancer, diabetes mellitus, rheumatologic and collagen-vascular disease, and gout; clinical signs of effusion in the knee, hotness or redness at the knee, history of trauma to the knee; history of knee arthroplasty or other surgeries; BMI $>42$; presence of active radiculopathy, myopathy, or peripheral neuropathy in lower limbs; pregnancy or breastfeeding; allergy to piroxicam and other NSAIDs; severe gastrointestinal, hepatic, renal, respiratory, cerebral, and cardiovascular diseases; severe KOA in X-ray (grade 4 of the Kellgren-Lawrence scale); any condition with bleeding tendency; administration of anticoagulant drugs; any serious systemic or local infection (such as brucellosis); inability to complete the questionnaires.

\subsection{Intervention}

2.4.1. Mesotherapy Group. Patients received subcutaneous injection by using the disposable sterile syringes (thin needle: $0.27 \mathrm{~mm} \times 4 \mathrm{~mm}$ ) containing a mix of $20 \mathrm{mg} / 1 \mathrm{ml}$ piroxicam (Roxicam ${ }^{\mathrm{R}}$, manufactured by OSVAH Pharma. Co., Tehran, Iran) and $2 \mathrm{ml}$ of $2 \%$ lidocaine $\left(X_{y l e x}{ }^{\mathrm{R}}\right.$, manufactured by Sinadarou Pharma. Co., Tehran, Iran) in painful points around the knee (2-6 points). The needle angle when entering the skin was 30-45 degrees. Before the injections, the skin was prepped and draped. After 10 days of injection, it was repeated (each patient received two sessions of mesotherapy). The patients were trained for lifestyle modification and quadriceps femoris strengthening exercise (with contraction of the muscle) and hamstring and calve muscles stretching (with straight leg rising to 60-70 degrees) [25]. Duration of each exercise was 15-30 seconds and repeated 10-30 times a day according to the patient tolerance. Patients were advised to avoid any other treatment for KOA.

2.4.2. Oral Administration Group. Patients received an oral administration of 2 capsules of piroxicam (piroxicam $10 \mathrm{mg}$, manufactured by Zahravi Pharma. Co., Tabriz, Iran) once 


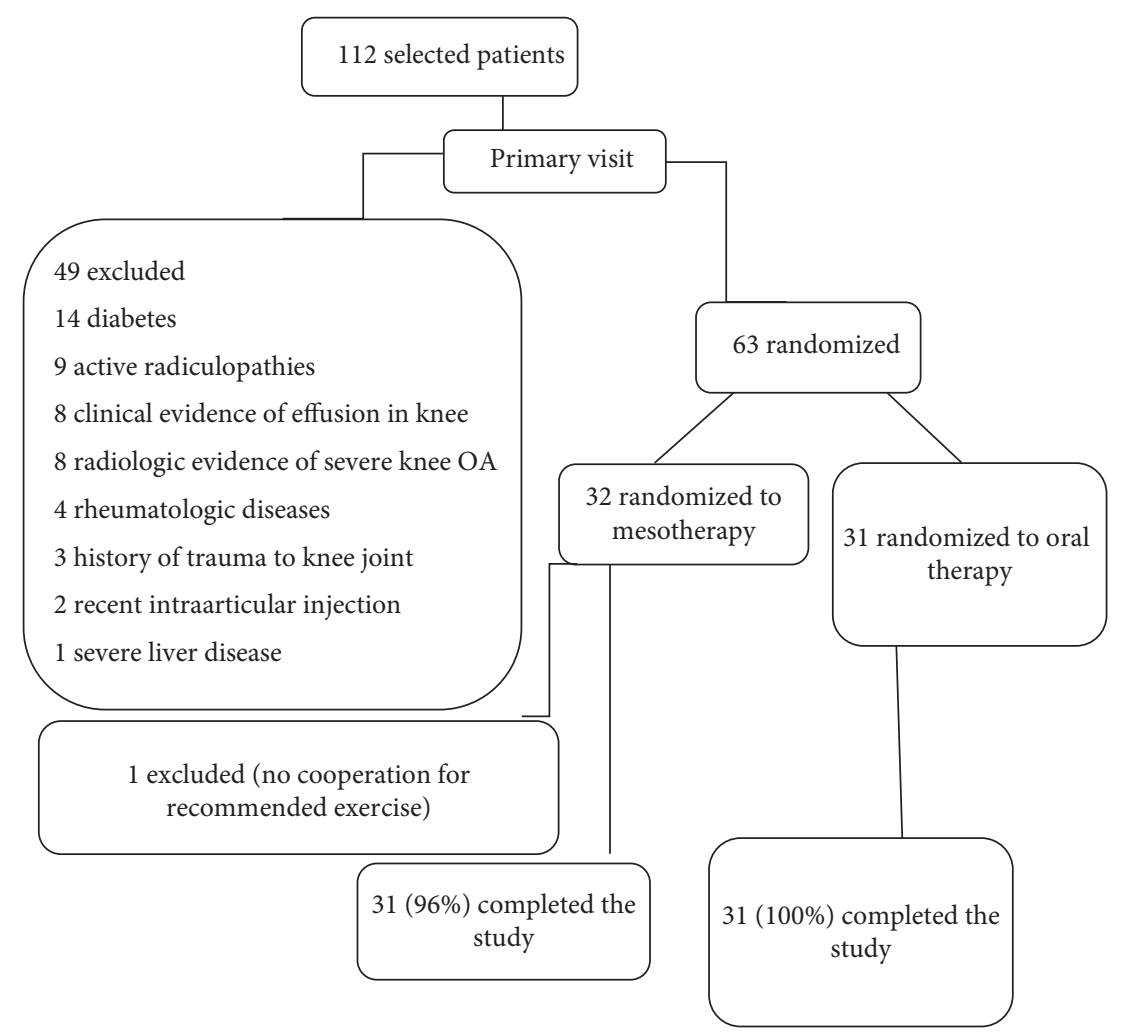

FIgURE 1: Study consort flowchart.

every day for 10 days. All patients were trained for lifestyle modification and the same exercises as the mesotherapy group. Patients were advised to avoid any other treatment for KOA.

All patients in both groups were given a phone number to ask any probable question. The treatment complications were considered in each follow-up.

2.5. Outcomes. All patients were evaluated before treatment and 2, 4, and 8 weeks after treatment by the Visual Analogue Scale (VAS), Western Ontario and McMaster Universities Arthritis Index (WOMAC), and Oxford Knee Scale (OKS) questionnaires. VAS evaluates the pain intensity with 10 degrees from 0 (no pain) to 10 (most possible pain) [24]. WOMAC is an index for evaluation of function with three parts: pain with five items, joint stiffness with 2 items, and physical function with 17 items (total WOMAC score was 24 items). Each item includes 5 scales from 0 to 4 (none: 0 , mild: 1 , moderate: 2 , severe: 3 , and extreme: 4). The total WOMAC score is defined between 0 as the best function and 96 as the worst function [26]. Also, OKS evaluates the function of the patient and contains 12 items with 5 scores from 0 to 4 (none: 4 , very mild: 3 , mild: 2 , moderate: 1 , and severe: 0 ); score 0 points to the worst function and score 48 refers to the best performance [27]. According to previous studies, there is no limitation to the use of questionnaires for one person's knees $[26,27]$. The most important demographic criteria were age, sex, and body mass index (BMI).

2.6. Sample Size. The sample size was determined based on the statistical analysis considering data from similar studies with a significance level of $0.5(p<0.05)$, confidence interval of $95 \%$, power of 1.20 , and probable dropout rate of $20 \%$. The number of samples in each group was 30 (each knee was considered as a sample).

2.7. Randomization and Blinding. 63 patients were randomly divided into two parallel groups (group A: mesotherapy and group B: oral therapy). We used restricted block randomization. The statistician was unaware of allocation (singleblind study).

2.8. Statistical Method. All data were analyzed using SPSS (Statistical Package for the Social Science) software version. 22. Normality of the data was evaluated by Shapiro-Wilk's test. To evaluate the changes in data over the time, the Freidman test and the chi-square $\left(X^{2}\right)$ index were applied. To compare the two groups, we used the 
independent $t$-test. Standard deviation, mean, and confidence interval were calculated, and finally statistical significance was considered as a $p$ value $<0.05$. The Wilcoxon signal ranks test and Bonferroni correction were used for comparison of 6 time points (time 1: before treatment and $2^{\text {nd }}$ week after treatment, time 2 : before treatment and $4^{\text {th }}$ week after treatment, time 3 : before treatment and $8^{\text {th }}$ week after treatment, time $4: 2^{\text {nd }}$ and $4^{\text {th }}$ weeks after treatment, time 5: $2^{\text {nd }}$ and $8^{\text {th }}$ weeks after treatment, and finally time $6: 4^{\text {th }}$ and $8^{\text {th }}$ weeks after treatment) in each group, and the Bonferroni-corrected $p$ value was considered as $<0.008(0.05 \div 6)$.

2.9. Ethical Considerations. We explained both methods and side effects to each patient before participating in the study. All patients gave their informed consent form. They could withdraw from the study whenever they wanted. All patients were trained on lifestyle modification and appropriate exercise for KOA (standard and basic treatments). All complications were carefully followed.

This study was approved by the Medical Ethics Committee of the Shiraz University of Medical Sciences (SUMS) with the ethics number: IR.SUMS.MED.REC.1395.59 and registered with a registration ID of $2017052434113 \mathrm{~N} 1$ at the IRCT (Iranian Registry of Clinical Trials).

\section{Results}

We assessed 112 patients with knee pain who were the candidates to participate in our study. Forty-nine patients had at least one exclusion criterion and were not included. Other patients $(n=63)$ were randomized to two groups: mesotherapy (group A) and oral therapy (group B). One patient from the mesotherapy group withdrew from the study. Finally, 31 patients in the mesotherapy group and 31 in the oral therapy group completed the study. There was no statistically significant difference in age, sex, BMI (Table 1), VAS, OKS, and WOMAC scores before the treatment (Table 2).

The VAS score was decreased in both groups after 2, 4, and 8 weeks of follow-up ( $p$ value $<0.001$ ). There was no significant difference between the two groups at any time ( $p$ value $>0.05$ ) (Table 3 ), but pain decrement compared to the baseline was more in the mesotherapy group in the fourth week ( $p$ value $<0.001)$ (Table 4$)$. Data analysis showed that the OKS score improved in both groups after 2,4 , and 8 weeks of follow-up ( $p$ value $<0.05$ ), but this increment was more in the mesotherapy group ( $p$ value $<0.05$ ) (Table 3 ). For both the VAS score and the OKS score, at all times except time 6 in the mesotherapy group and time 4 in the oral group, the Bonferronicorrected $p$ value was $<0.008$. These results were also applied to the change in function compared to the baseline (Table 4). The results of the WOMAC analysis showed that pain and function and total score improved significantly in both groups after 2,4 , and 8 weeks of follow-up ( $p$ value $<0.05$ ) with better results in the mesotherapy group ( $p$ value $<0.05$ ), but improvement in the joint stiffness occurred only in the mesotherapy group ( $p$ value $<0.001$ ) (Table 5 ). Although changes in pain, function, and the total WOMAC score, compared to the baseline, were clearly observed in both groups ( $p$ value $<0.05)$, the changes in the mesotherapy group were more significantly different $(p$ value $<0.001)$ (Table 6). For the total WOMAC score, at all times except time 6 in the mesotherapy group, the Bonferroni-corrected $p$ value was $<0.008$.

\section{Discussion}

There are important limitations for systemic therapy in patients with KOA, especially in the elderly. In this study, we evaluated the effectiveness of mesotherapy with piroxicam (as a local treatment) on pain reduction and functional improvement in patients with KOA and compared it with oral piroxicam prescription. The study's results showed that mesotherapy with piroxicam can reduce the pain similar to administration of oral piroxicam at 8 weeks of follow-up. Both methods improved the function (OKS and WOMAC scales) in this time, but effects were better in the mesotherapy group (perhaps due to higher drug concentration at the local area in the mesotherapy procedure). There was no significant difference in VAS, OKS, and WOMAC scores between the fourth and eighth weeks after treatment in the mesotherapy group. In the oral group, this was true for VAS and OKS scores between the second and fourth weeks after treatment. In our study, there were no serious side effects. In the oral therapy group, 8 patients (10 knees, $32.2 \%$ of the total subjects) had heart burn which disappeared with 3 doses of ranitidine for 7 days. Pain at the injection site is one of the most common complications of mesotherapy. In our study, one patient (3.2\% of the total subjects) reported pain for one day at the injection site that improved with ice massage and 2 doses of $500 \mathrm{mg}$ acetaminophen. Bruises were created at the injection site on 12 knees $(38.7 \%$ of the total subjects) that resolved to a maximum of 4 weeks. Complications such as bleeding or hypersensitivity reactions were not observed.

In another study on the efficacy and safety of mesotherapy in patients with KOA (published in 2018) conducted by Chen et al., it was shown that oral therapy with diclofenac ( $75 \mathrm{mg}$ twice per day for 3 months) and mesotherapy with 2 protocols for acute ( $40 \mathrm{mg}$ piroxicam, 100 units of calcitonin, and $2 \mathrm{cc}$ lidocaine $1 \%$ ) and chronic conditions ( $2 \mathrm{cc}$ procaine $2 \%, 2 \mathrm{cc}$ organic silica, and 100 units of calcitonin) significantly improved biochemical markers and clinical conditions. Mesotherapy had significantly fewer side effects and was more efficient in terms of hematology and WOMAC scores [17]. Saggini et al. conducted a study in 2015 to compare the efficacy of mesotherapy with $25 \mathrm{mg}$ diclofenac, nine 
TABLE 1: Baseline characteristics of patients.

\begin{tabular}{lccc}
\hline & Mesotherapy group & Oral therapy group & $p$ value \\
\hline Sex & $\mathrm{F}^{\mathrm{a}}: 29(93.5 \%)$ & F: 25 $(80.6 \%)$ & \\
& $\mathrm{M}^{\mathrm{b}}: 2(6.5 \%)$ & M: $6(19.4 \%)$ & 0.09 \\
Age, mean $\pm \mathrm{SD}^{\mathrm{c}}$ & $56.87 \pm 9.14$ & $53.45 \pm 8.63$ & 0.66 \\
$\mathrm{BMI}^{\mathrm{d}}\left(\mathrm{kg} / \mathrm{m}^{2}\right)$, mean $\pm \mathrm{SD}$ & $27.61 \pm 5.08$ & $28.3 \pm 4.66$ & 0.15 \\
\hline
\end{tabular}

${ }^{\mathrm{a}} \mathrm{F}$, female; ${ }^{\mathrm{b}} \mathrm{M}$, male; ${ }^{\mathrm{c}} \mathrm{SD}$, standard deviation; ${ }^{\mathrm{d}} \mathrm{BMI}$, body mass index.

TABLE 2: Baseline VAS, OKS, and WOMAC scores.

\begin{tabular}{lccc}
\hline & Mesotherapy group & Oral therapy group & $p$ value \\
\hline VAS $^{\mathrm{a}}$, mean $\pm \mathrm{SD}^{\mathrm{b}}$ & $8.25 \pm 1.99$ & $7.45 \pm 1.68$ & 0.07 \\
$\mathrm{OKS}^{\mathrm{c}}$, mean $\pm \mathrm{SD}$ & $22.93 \pm 7.57$ & $21.13 \pm 4.54$ & \\
WOMAC & & & \\
Pain & & & \\
Stiffness & $16.0 \pm 3.49$ & $3.96 \pm 3.33$ & 0.13 \\
Function & $4.03 \pm 1.94$ & $3.97 \pm 1.34$ & $0.61 \pm 9.75$ \\
Total score & $33.35 \pm 9.85$ & $50.44 \pm 14.14$ & 0.12 \\
\hline
\end{tabular}

${ }^{\mathrm{a}}$ VAS, Visual Analogue Scale; ${ }^{\mathrm{b}} \mathrm{SD}$, standard deviation; ${ }^{\mathrm{c}} \mathrm{OKS}$, Oxford Knee Scale; ${ }^{\mathrm{d}}$ WOMAC, Western Ontario and McMaster Universities Arthritis Index.

TABLE 3: VAS and OKS scores in both groups during the follow-up.

\begin{tabular}{|c|c|c|c|c|}
\hline & Mesotherapy group & Oral therapy group & $p$ value (within groups) & $p$ value (between groups) \\
\hline $\mathrm{VAS}^{\mathrm{a}}$, mean, $\mathrm{SD}^{\mathrm{b}}$ & & & 0.001 & \\
\hline Baseline & $8.25 \pm 1.99$ & $7.45 \pm 1.68$ & & 0.07 \\
\hline $2^{\text {nd }}$ week & $5.25 \pm 1.99$ & $5.22 \pm 2.27$ & & 0.95 \\
\hline $4^{\text {th }}$ week & $3.51 \pm 2.17$ & $4.48 \pm 2.06$ & & 0.07 \\
\hline $8^{\text {th }}$ week & $3.12 \pm 2.09$ & $3.38 \pm 2.02$ & & 0.62 \\
\hline $\mathrm{OKS}^{\mathrm{c}}$, mean $\pm \mathrm{SD}$ & & & $\begin{array}{l}\text { Mesotherapy group: } 0.001 \\
\text { Oral therapy group: } 0.04\end{array}$ & \\
\hline Baseline & $22.93 \pm 7.57$ & $21.13 \pm 4.54$ & & 0.11 \\
\hline $2^{\text {nd }}$ week & $31.60 \pm 8.28$ & $25.33 \pm 5.93$ & & 0.03 \\
\hline $4^{\text {th }}$ week & $38.41 \pm 9.12$ & $27.25 \pm 6.99$ & & 0.01 \\
\hline $8^{\text {th }}$ week & $39.05 \pm 15.32$ & $30.22 \pm 8.10$ & & 0.01 \\
\hline
\end{tabular}

${ }^{\mathrm{a}}$ VAS, Visual Analogue Scale; ${ }^{\mathrm{b}} \mathrm{SD}$, standard deviation; ${ }^{\mathrm{c} O K S}$, Oxford Knee Scale.

TABLE 4: VAS score decrement and OKS score increment in both groups during the follow-up compared to the baseline.

\begin{tabular}{|c|c|c|c|c|}
\hline & Mesotherapy group & Oral therapy group & $p$ value (within groups) & $p$ value (between groups) \\
\hline $\mathrm{VAS}^{\mathrm{a}}$ decrement, mean $\pm \mathrm{SD}^{\mathrm{b}}$ & & & 0.001 & \\
\hline In 2 weeks & $3.00 \pm 1.86$ & $2.22 \pm 2.24$ & & 0.14 \\
\hline In 4 weeks & $4.74 \pm 2.26$ & $2.96 \pm 1.45$ & & 0.001 \\
\hline In 8 weeks & $5.12 \pm 2.49$ & $4.06 \pm 2.76$ & & 0.11 \\
\hline $\mathrm{OKS}^{\mathrm{c}}$ increment, mean $\pm \mathrm{SD}$ & & & $\begin{array}{l}\text { Mesotherapy group: } 0.001 \\
\text { Oral therapy group: } 0.03\end{array}$ & \\
\hline In 2 weeks & $8.67 \pm 5.00$ & $4.19 \pm 5.49$ & & 0.001 \\
\hline In 4 weeks & $15.48 \pm 7.11$ & $6.12 \pm 5.53$ & & 0.001 \\
\hline In 8 weeks & $16.12 \pm 9.39$ & $9.09 \pm 7.02$ & & 0.001 \\
\hline
\end{tabular}

${ }^{\mathrm{a}}$ VAS, Visual Analogue Scale; ${ }^{\mathrm{b}} \mathrm{SD}$, standard deviation; ${ }^{\mathrm{c} O K S}$, Oxford Knee Scale.

sessions, and oral diclofenac (50 $\mathrm{mg}$ once a day for 3 weeks) on pes anserine bursitis in KOA (grade 2 of Kellgren-Lawrence classification). In all 112 patients, pain level decreased significantly after treatment. Ultrasonography showed a reduction in the hypoechoic area related to bursitis only in the mesotherapy group [20].

There are studies that support the good effect of mesotherapy in reducing back pain $[19,22]$.
In our study, sessions of mesotherapy were limited (only 2 sessions) and oral piroxicam was prescribed only for 10 days $(20 \mathrm{mg}$ once a day). This study showed that mesotherapy was an effective and safe procedure in patients with mild or moderate KOA.

The limitations of our study included the lack of objective evaluation of the effects of interventions, short-term follow-up, lack of measurement of drug plasma level, and lack of a placebo group. 
TABLE 5: WOMAC score in both groups during the follow-up.

\begin{tabular}{|c|c|c|c|c|}
\hline & Mesotherapy group & Oral therapy group & $p$ value (within groups) & $p$ value (between groups) \\
\hline \multicolumn{5}{|c|}{ WOMAC $^{\mathrm{a}}$, mean $\pm \mathrm{SD}^{\mathrm{b}}$} \\
\hline Pain & & & $\begin{array}{l}\text { Mesotherapy group: } 0.001 \\
\text { Oral therapy group: } 0.04\end{array}$ & \\
\hline Baseline & $16.00 \pm 3.49$ & $14.96 \pm 3.33$ & & 0.13 \\
\hline $2^{\text {nd }}$ week & $11.13 \pm 2.89$ & $13.26 \pm 3.01$ & & 0.04 \\
\hline $4^{\text {th }}$ week & $8.04 \pm 2.40$ & $12.64 \pm 2.98$ & & 0.01 \\
\hline $8^{\text {th }}$ week & $7.55 \pm 2.40$ & $11.38 \pm 2.90$ & & 0.01 \\
\hline Stiffness & & & $\begin{array}{l}\text { Mesotherapy group: } 0.001 \\
\text { Oral therapy group: } 0.06\end{array}$ & \\
\hline Baseline & $4.03 \pm 1.94$ & $3.97 \pm 1.34$ & & 0.66 \\
\hline $2^{\text {nd }}$ week & $3.07 \pm 1.23$ & $3.78 \pm 1.25$ & & 0.03 \\
\hline $4^{\text {th }}$ week & $2.36 \pm 0.61$ & $3.68 \pm 1.15$ & & 0.01 \\
\hline $8^{\text {th }}$ week & $2.33 \pm 0.65$ & $3.36 \pm 1.05$ & & 0.02 \\
\hline Function & & & $\begin{array}{l}\text { Mesotherapy group: } 0.001 \\
\text { Oral therapy group: } 0.03\end{array}$ & \\
\hline Baseline & $33.35 \pm 9.85$ & $31.51 \pm 9.75$ & & 0.12 \\
\hline $2^{\text {nd }}$ week & $18.32 \pm 8.19$ & $24.68 \pm 8.11$ & & 0.04 \\
\hline $4^{\text {th }}$ week & $9.45 \pm 6.48$ & $16.45 \pm 8.09$ & & 0.03 \\
\hline $8^{\text {th }}$ week & $8.74 \pm 7.07$ & $12.63 \pm 7.78$ & & 0.04 \\
\hline Total score & & & $\begin{array}{l}\text { Mesotherapy group: } 0.001 \\
\text { Oral therapy group: } 0.04\end{array}$ & \\
\hline Baseline & $53.38 \pm 14.41$ & $50.44 \pm 14.14$ & & 0.17 \\
\hline $2^{\text {nd }}$ week & $32.52 \pm 10.11$ & $41.72 \pm 10.76$ & & 0.02 \\
\hline $4^{\text {th }}$ week & $19.85 \pm 6.78$ & $32.77 \pm 10.34$ & & 0.001 \\
\hline $8^{\text {th }}$ week & $18.62 \pm 6.11$ & $27.37 \pm 9.55$ & & 0.02 \\
\hline
\end{tabular}

${ }^{a}$ WOMAC, Western Ontario and McMaster Universities Arthritis Index; ${ }^{b} \mathrm{SD}$, standard deviation.

TABLE 6: WOMAC score improvement in both groups during the follow-up compared to the baseline.

\begin{tabular}{|c|c|c|c|c|}
\hline & Mesotherapy group & Oral therapy group & $p$ value (within groups) & $p$ value (between groups) \\
\hline \multicolumn{5}{|c|}{ WOMAC $^{\mathrm{a}}$ improvement, mean $\pm \mathrm{SD}^{\mathrm{b}}$} \\
\hline Pain decrement & & & $\begin{array}{l}\text { Mesotherapy group: } 0.001 \\
\text { Oral therapy group: } 0.04\end{array}$ & \\
\hline In 2 weeks & $4.87 \pm 3.05$ & $1.70 \pm 2.84$ & & 0.001 \\
\hline In 4 weeks & $7.96 \pm 3.19$ & $2.32 \pm 2.84$ & & 0.001 \\
\hline In 8 weeks & $8.45 \pm 4.28$ & $3.58 \pm 3.37$ & & 0.001 \\
\hline Stiffness decrement & & & $\begin{array}{l}\text { Mesotherapy group: } 0.001 \\
\text { Oral therapy group: } 0.06\end{array}$ & \\
\hline In 2 weeks & $0.96 \pm 1.16$ & $0.19 \pm 0.54$ & & 0.001 \\
\hline In 4 weeks & $1.67 \pm 1.72$ & $0.29 \pm 0.58$ & & 0.001 \\
\hline In 8 weeks & $1.70 \pm 1.69$ & $0.61 \pm 1.17$ & & 0.004 \\
\hline Function improvement & & & $\begin{array}{c}\text { Mesotherapy group: } 0.001 \\
\text { Oral therapy group: } 0.03\end{array}$ & \\
\hline In 2 weeks & $15.03 \pm 8.59$ & $6.83 \pm 9.82$ & & 0.001 \\
\hline In 4 weeks & $23.90 \pm 10.03$ & $15.06 \pm 8.60$ & & 0.001 \\
\hline In 8 weeks & $24.61 \pm 12.25$ & $18.88 \pm 9.52$ & & 0.001 \\
\hline Total score improvement & & & $\begin{array}{l}\text { Mesotherapy group: } 0.001 \\
\text { Oral therapy group: } 0.04\end{array}$ & \\
\hline In 2 weeks & $20.86 \pm 11.93$ & $8.72 \pm 4.36$ & & 0.001 \\
\hline In 4 weeks & $33.53 \pm 13.96$ & $17.67 \pm 7.11$ & & 0.001 \\
\hline In 8 weeks & $34.76 \pm 17.14$ & $23.07 \pm 10.52$ & & 0.001 \\
\hline
\end{tabular}

${ }^{\mathrm{a}}$ WOMAC, Western Ontario and McMaster Universities Arthritis Index; ${ }^{\mathrm{b}} \mathrm{SD}$, standard deviation.

\section{Conclusion}

Mesotherapy is an effective and well-tolerated method for pain reduction and function improvement in patients with mild-tomoderate KOA in the short term and can be considered as an alternative therapy in patients with contraindications for systemic therapy with NSAIDs. These results are based on subjective findings. Further studies are required to be conducted to evaluate mesotherapy with different methods, long-term follow-up, and objective findings. 


\section{Data Availability}

The data used to support the findings of this study are available from the corresponding author upon request.

\section{Disclosure}

This study is a part of the thesis of Fahime Estakhri (Grant no: 95-01-01-11896) with the ethics number: IR. SUMS. MED. REC. 1395.59.

\section{Conflicts of Interest}

There are no conflicts of interest to be mentioned.

\section{Authors' Contributions}

All authors had equal contribution in this trial.

\section{Acknowledgments}

The authors would like to thank the Shiraz University of Medical Sciences, Shiraz, Iran, and also the Center for Development of Clinical Research of Nemazee Hospital and Dr. Nasrin Shokrpour and Dr. Faghih for editorial assistance and statistical analysis.

\section{References}

[1] J. W. Bijlsma, F. Berenbaum, and F. P. Lafeber, "Osteoarthritis: an update with relevance for clinical practice," The Lancet, vol. 377, no. 9783, pp. 2115-2126, 2011.

[2] R. C. Lawrence, D. T. Felson, C. G. Helmick et al., "Estimates of the prevalence of arthritis and other rheumatic conditions in the United States. part II," Arthritis Rheum, vol. 58, no. 1, pp. 23-26, 2008.

[3] L. M. March and C. J. M. Bachmeier, "10 Economics of osteoarthritis: a global perspective," Baillière's Clinical Rheumatology, vol. 11, no. 4, pp. 817-834, 1997.

[4] W. F. Stewart, J. A. Ricci, E. Chee, D. Morganstein, and R. Lipton, "Lost productive time and cost due to common pain conditions in the US workforce," JAMA, vol. 290, no. 18, pp. 2443-2454, 2003.

[5] S. A. Raeissadat, S. M. Rayegani, H. Hassanabadi et al., "Knee osteoarthritis injection choices: platelet-rich plasma (PRP) versus hyaluronic acid (a one-year randomized clinical trial)," Clin Med Insights Arthritis Musculoskeletal Disord, vol. 8, pp. 1-8, 2015.

[6] J. Kielly, E. M. Davis, and C. Marra, "Practice guidelines for pharmacists: the management of osteoarthritis," Canadian Pharmacists Journal/Revue des Pharmaciens du Canada, vol. 150, no. 3, pp. 156-168, 2017.

[7] H. R. Farpour and F. Fereydooni, "Comparative effectiveness of intra-articular prolotherapy versus peri-articular prolotherapy on pain reduction and improving function in patients with knee osteoarthritis: a randomized clinical trial," Electronic Physician, vol. 9, no. 11, pp. 5663-5669, 2017.

[8] M. C. Hochberg, R. D. Altman, K. T. April et al., "American college of rheumatology 2012 recommendations for the use of nonpharmacologic and pharmacologic therapies in osteoarthritis of the hand, hip, and knee," Arthritis Care \& Research, vol. 64, no. 4, pp. 465-474, 2012.
[9] G. C. Machado, C. G. Maher, P. H. Ferreira et al., "Efficacy and safety of paracetamol for spinal pain and osteoarthritis: systematic review and meta-analysis of randomized placebo controlled trials," BMJ, vol. 350, no. mar 312, 2015.

[10] J. Flood, "The role of acetaminophen in the treatment of osteoarthritis," The American Journal of Managed Care, vol. 16, no. 54, pp. 548-554, 2010.

[11] P. Guyot, S. Pandhi, R. M. Nixon, A. Iqbal, R. L. Chaves, and R. A. Moore, "Efficacy and safety of diclofenac in osteoarthritis: results of a network meta-analysis of unpublished legacy studies," Scandinavian Journal of Pain, vol. 16, no. 1, pp. 74-88, 2017.

[12] E. E. Krebs, A. Gravely, S. Nugent et al., "Effect of opioid vs nonopioid medications on pain-related function in patients with chronic back pain or hip or knee osteoarthritis pain," JAMA, vol. 319, no. 9, pp. 872-882, 2018.

[13] S. M. Kroenke, S. P. Chornobai, S. P. Chornobai, Y. L. Holyuk, and G. G. Bondariev, "Modern approach to conservative treatment of degenerative diseases of joints using regenerative medicine techniques," Problems of Cryobiology and Cryomedicine, vol. 28, no. 1, pp. 079-083, 2018.

[14] F. Buttgereit, G. R. Burmester, and J. W. Bijlsma, "Nonsurgical management of knee osteoarthritis: where are we now and where do we need to go?," RMD Open, vol. 1, no. 1, Article ID e000027, 2015.

[15] C. Sostres, C. J. Gargallo, M. T. Arroyo, and A. Lanas, "Adverse effects of non-steroidal anti-inflammatory drugs (NSAIDs, aspirin and coxibs) on upper gastrointestinal tract," Best Practice \& Research Clinical Gastroenterology, vol. 24, no. 12, pp. 121-132, 2010.

[16] Y. Grag, J. Singh, H. S. Sohal, R. Gore, and A. Kumar, "Comparison of clinical effectiveness and safety of newer nonsteroidal anti-inflammatory drugs in patients of osteoarthritis of knee joint: a randomized, prospective, open-label parallel-group study," Indian Journal of Pharmacology, vol. 49, no. 5, pp. 383-389, 2017.

[17] L. Chen, D. Li, J. Zhong, B. QIU, and X. Wu, "Therapeutic effectiveness and safety of mesotherapy in patients with osteoarthritis of the knee," Evidence-Based Complementary and Alternative Medicine, vol. 2018, Article ID 6513049, 6 pages, 2018.

[18] D. M. Hexsel and J. D. Fernandes, "Complications of mesotherapy," Management of Complications of Cosmetic Procedures, Springer, Berlin, Germany, 2012.

[19] M. Mammucari, A. Gatti, S. Maggiori, and A. F. Sabato, "Role of mesotherapy in musculoskeletal pain: opinions from the Italian society of mesotherapy," Evidence-Based Complementary and Alternative Medicine, vol. 2012, Article ID 436959, 12 pages, 2012.

[20] R. Saggini, A. Di Stefano, I. Dodaj, L. Scarcello, and R. G. Bellomo, "Pes anserine bursitis in symptomatic osteoarthritis patients: a mesotherapy treatment study," The Journal of Alternative and Complementary Medicine, vol. 21, no. 8, pp. 480-484, 2015.

[21] M. Mammucari, E. Maggiori, M. Lazzari, and S. Natoli, "Should the general practitioner consider mesotherapy (intradermal therapy) to manage localized pain?," Pain and Therapy, vol. 5, no. 1, pp. 123-126, 2016.

[22] C. Costantino, E. Marangio, and G. Coruzzi, "Mesotherapy versus systemic therapy in the treatment of acute low back pain: a randomized trial," Evidence-Based Complementary and Alternative Medicine, vol. 2011, Article ID 317183, 6 pages, 2011.

[23] J. H. Kellgren and J. S. Lawrence, "Radiological assessment of osteo-arthrosis," Annals of the Rheumatic Diseases, vol. 16, no. 4, pp. 494-502, 1957. 
[24] D. D. Price, P. A. McGrath, A. Rafii, and B. Buckingham, "The validation of visual analogue scales as ratio scale measures for chronic and experimental paIN," Pain, vol. 17, no. 1, pp. $45-56,1983$.

[25] C. T. Kuru, B. Kavlak, O. Aydogdu et al., "The effects of therapeutic exercises on pain, muscle strength, functional capacity,balance and hemodynamic parameters in knee osteoarthritis patients:a randomized controlled study of supervised versus home exercises," Rheumatologic Internist, vol. 37, no. 3, pp. 399-407, 2017.

[26] M. H. Ebrahimzade, H. Makhmalbaf, A. S. Birjandinejad, and S. H. Soltani-Moghaddas, "Cross-cultural adaptation and validation of the Persian version of the Oxford knee score in patients with knee osteoarthritis," DescriptionThe Iranian Journal of Medical Sciences, vol. 39, no. 6, pp. 529-535, 2014.

[27] B. Eftekhar-Sadat, S. H. Niknejad-Hosseyni, A. BabaeiGhazani, V. Toopchizadeh, and H. Sadeghi, "Reliability and validity of Persian version of Western Ontario and McMaster Universities Osteoarthritis index in knee osteoarthritis," Journal of Analytical Research in Clinical Medicine, vol. 3, no. 3, pp. 170-177, 2015. 